\title{
Acute Liver Failure
}

\author{
William M. Lee, M.D., F.A.C.P. ${ }^{1}$ \\ ${ }^{1}$ Division of Digestive and Liver Diseases, Department of Internal \\ Medicine, University of Texas Southwestern Medical Center at Dallas, \\ Dallas, Texas. \\ Semin Respir Crit Care Med 2012;33:36-45.
}

Address for correspondence and reprint requests William M. Lee, M.D., Division of Digestive and Liver Diseases, Department of Internal Medicine, University of Texas Southwestern Medical Center, 5959 Harry Hines Blvd. HP4. 420E, Dallas, TX 75390-8887

(e-mail: william.lee@utsouthwestern.edu).

\begin{abstract}
\section{Keywords}

- acute liver injury

- acetaminophen toxicity

- coagulopathy

- encephalopathy

- hepatic necrosis

Acute liver failure (ALF) (sometimes referred to as fulminant hepatic failure) is a clinical syndrome from a variety of causes resulting from rapid loss in hepatocyte function, typically associated with coagulopathy and encephalopathy in a patient without preexisting liver disease or cirrhosis. Cerebral edema is a cardinal feature and may produce uncal herniation, yielding brain stem compression and death. The typical interval from onset of symptoms to onset of encephalopathy is 1 to 2 weeks, but cases evolving more slowly, up to 6 months, may still be included in the definition. ALF is rare, affecting 2000 patients annually in the United States, and comprises $~ 7 \%$ of liver transplants annually. Currently, in the United States, acetaminophen accounts for $\sim 50 \%$ of all cases of ALF, but other etiologies include hepatitis, drug-induced liver injury, autoimmune hepatitis. Prior to the availability of liver transplantation (LT), mortality of ALF was extremely high, often exceeding $90 \%$; most common causes of death were multiorgan failure, hemorrhage, infection, and cerebral edema. Fortunately, survival has improved considerably in the last 3 decades (overall survival now exceeds 60\%). In large part, this improved survival reflects the option of LT but also reflects the high frequency of acetaminophen toxicity as a cause of ALF. In fact, most patients with ALF are not candidates for LT. Critical care of patients with ALF is key to their survival, and decisions must sometimes be made with inadequate information. We review standard practices (medical, pharmacological, and LT) and new research initiatives and findings for this interesting but vexing orphan disease. Particular attention will be paid to practical matters for clinicians to consider in approaching the ALF patient.
\end{abstract}

Acute liver failure (ALF) is the most common term applied to an unusual clinical syndrome resulting from rapid loss in hepatocyte function. It occurs infrequently, affecting 2000 patients annually in the United States, and comprises $~ 7 \%$ of liver transplants annually. ALF is the culmination of severe liver cell injury from a variety of different causes, but it leads to a relatively uniform clinical syndrome characterized by encephalopathy and coagulopathy, the hallmark features. ${ }^{1}$ Because of its rarity, research in ALF has been limited to a handful of large units or to collaborative networks such as the National Institutes of Health (NIH)-sponsored U.S. Acute Liver Failure Study Group (ALFSG).

This review provides an overview of standard practices and new research initiatives and findings for this interesting but vexing orphan disease. Particular attention is paid to practical matters for clinicians to consider in approaching the ALF patient. Patients with ALF are supremely ill; the condition carries a high mortality rate, and transplantation, while lifesaving, is not always an option. Critical care of these rare patients is key to their survival, and decisions must sometimes be made with inadequate information. Experience is probably a great teacher here, although ALF's rarity means that few have in-depth experience with ALF patients.

\section{Definition}

Acute liver failure (sometimes referred to as fulminant hepatic failure) is most commonly defined as the onset of
Issue Theme Pulmonary and Critical Care Considerations in Hepatic Disease; Guest Editors, Tisha S. Wang, M.D. and Michael B. Fallon, M.D.
Copyright (C) 2012 by Thieme Medical Publishers, Inc., 333 Seventh Avenue, New York, NY 10001, USA. Tel: +1(212) 584-4662.
DOI http://dx.doi.org/ 10.1055/s-0032-1301733. ISSN 1069-3424. 
coagulopathy [international normalized ratio (INR) $\geq 1.5$ ] and encephalopathy (any degree of altered mentation) in a patient without preexisting liver disease or cirrhosis. ${ }^{1}$ The typical interval from onset of symptoms to onset of encephalopathy is 1 to 2 weeks, but cases evolving more slowly, up to 6 months, may still be included in the definition. A clinical feature that is virtually unique to ALF is cerebral edema, swelling of the brain, that may produce herniation of the uncus through the falx cerebrum, yielding brain stem compression and death. ${ }^{2}$ The morbidity and mortality of ALF recorded in small case series in the pretransplant era was extremely high, often exceeding $90 \%$, the causes of death including multiorgan failure, hemorrhage, infection, and cerebral edema. Fortunately, these dire outcomes have diminished somewhat due to a change in the causes of ALF to more benign etiologies over the past 40 years as well as to the introduction of liver transplantation. ${ }^{3,4}$ Patients have been designated as hyperacute, acute, and subacute in presentation depending on the interval from onset of disease to onset of encephalopathy. Different etiologies typically have a specific time frame. For example, acetaminophen cases are virtually always hyperacute, whereas viral hepatitis, idiosyncratic drug reactions, or indeterminate cause cases demonstrate slower onset and evolution (acute or subacute). ${ }^{5}$ Whether there is a distinct difference in outcome based on the length of disease itself remains unclear; however, the etiologic diagnosis per se appears to be the strongest driver of outcome. ${ }^{2}$

\section{Diagnosis}

The diagnosis of ALF must be considered in anyone presenting with the recent onset of a hepatic illness where the prothrombin time/INR has become prolonged. Mental altera- tions are part of virtually all definitions and the changes may be subtle, initially including agitation and confusion but usually progressing to deeper coma grades. Recently, efforts to develop more robust measures to define early encephalopathy grades have been proposed but are still not widely used, and little has taken the place of the West Haven coma grade ${ }^{1-4}$ system. ${ }^{6}$ Any mental alteration in conjunction with a prolonged INR ( $>1.5$ ), qualifies the patient as having ALF, provided the person has had an illness of short duration and does not have cirrhosis. With nondescript presenting symptoms, the diagnosis is often missed by the initial medical contact. However, the combination of coagulopathy and encephalopathy is unique and is only seen in this setting. The presence of any degree of encephalopathy indicates a severe, life-threatening condition that requires immediate hospitalization. Patients are best managed within an intensive care setting, and specialty units devoted to acute liver failure are available in the United Kingdom. ${ }^{3}$ Rapid evaluation for transfer to a transplantation center and consideration for liver transplantation is mandatory once any degree of mental alteration occurs because disease progression is often very rapid once cognition is disturbed. ${ }^{7}$

ALF has a common clinical picture, regardless of the etiology, that represents the final pathway of acute organ failure, different from cirrhosis but specific to one organ, the liver. Patients appear to be relatively hypotensive and vasodilated with low systemic vascular resistance, a picture of multisystem failure that resembles in some ways that of gram-negative sepsis or end-stage liver disease patients; however, portal hypertension and ascites are usually absent except in subacute cases. Renal failure resembling hepatorenal syndrome may develop and is reversible with return of hepatic function. Infection is unusually common in ALF,

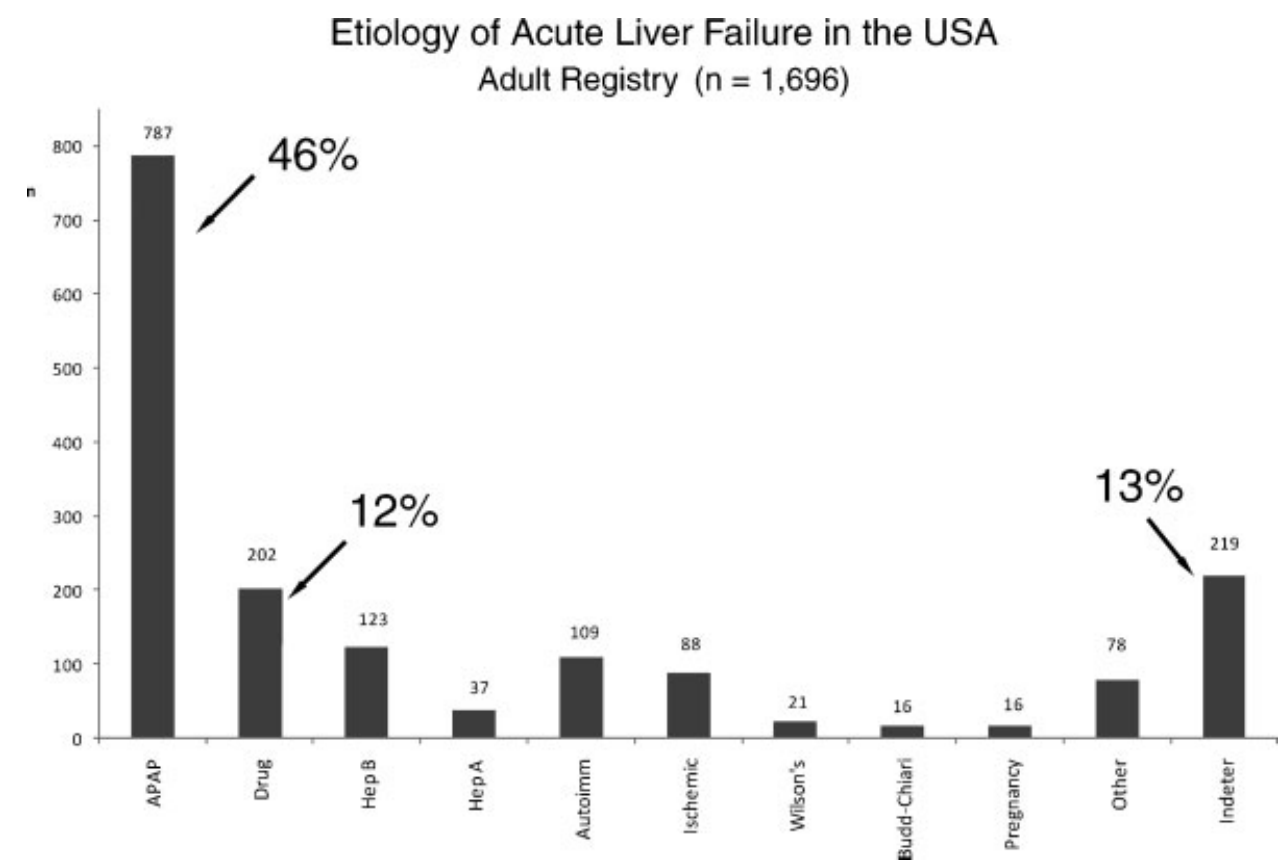

Figure 1 Bar graph depicting the percentage of 1696 patients enrolled in the Acute Liver Failure Study Group according to etiology. The highest number and percentage of patients are those due to acetaminophen. 
presumably signifying the role of the liver in the host's innate immune defenses.

\section{Etiology}

The causes of ALF are many and vary from country to country. In the era prior to transplantation, hepatitis $\mathrm{B}$ was very common and accounted for as many as 40 to $50 \%$ of US cases. Prior to 1990, although a well-recognized problem in the United Kingdom, acetaminophen did not account for more than a few US ALF cases. Current US figures provided by the ALFSG now show that acetaminophen-related ALF accounts for nearly $50 \%$ of all cases, with hepatitis B responsible for only $7 \%{ }^{4,8,9}$ The etiologic breakdown in the United States (-Fig. 1) is similar to that found in Europe but is far different in the developing world, such as India, where drug-induced liver injury is limited solely to isoniazid, and acetaminophen injury is virtually unknown. ${ }^{10}$

After acetaminophen, the most common causes are druginduced liver injury (comprising largely idiosyncratic reactions to prescription drugs), autoimmune hepatitis, and a variety of smaller groups. In many instances (15\%), the etiology remains unclear despite extensive history taking and laboratory assessment; these are termed indeterminate. Failure to obtain adequate history prior to onset of coma is one reason for missing the cause of illness in this setting. Regardless of the cause, the final common pathway is remarkably similar: worsening coma associated with a propensity for bleeding, infection, and renal failure leads to poor overall survival without transplantation.

\section{Acetaminophen-Related Liver Injury}

Although acetaminophen was approved for use in the 1950s, hepatotoxicity leading to liver failure was not recognized in significant numbers in the United States prior to 1980 . With the linking of aspirin to Reyes syndrome in children around 1980, Americans began turning to acetaminophen as a safer alternative for children and adults. Cases were reported during the 1980s of "therapeutic misadventures," and the association of unintended acetaminophen poisoning with alcohol was made. ${ }^{11}$ Although true incidence studies were (and are) not available, no large case series of ALF patients included acetaminophen until a retrospective study from the ALFSG, covering the period 1994 to 1996, found that 20\% of cases were related to acetaminophen. ${ }^{8}$ Similar data were reported in 2000 , in which $28 \%$ of transplant registry cases were believed related to acetaminophen during a 13-year retrospective study from the University of Pittsburgh. ${ }^{12}$ However, the US ALF study, in its first prospective analysis, recorded $39 \%$ of all ALF cases as due to acetaminophen between 1998 and 2001, ${ }^{3}$ increasing to 51\% in 2004. These figures represent the proportion of cases due to acetaminophen and may not be equated to actual incidence. Nevertheless, the increases are striking.

It is important to distinguish between considering all cases entering the hospital with presumed acetaminophen overdose and the smaller subset that experiences ALF. Of 71 acetaminophen overdose patients admitted to Parkland Hospital over a 39-month period, 50 were considered suicidal, with only one of 50 resulting in ALF and death, whereas six deaths occurred among 21 unintentional overdoses. ${ }^{13}$ Only the seven patients who died had developed acute hepatic failure, and only 10 of 50 suicidal patients had aminotransferase levels $\geq 1000 \mathrm{IU} / \mathrm{L}$. These data confirmed that most suicidal patients typically receive medical care within 4 hours of ingestion and are therefore reliably protected by the acetaminophen antidote, $N$-acetylcysteine (NAC). By contrast, overdoses that are called accidental, or more accurately unintentional, are associated with ingestion over several days, a specific cause of pain, and denial of suicidal intent. Late presentation is characteristic of the unintentional group because there is no understanding of possible harm; medical attention is sought only after symptoms of toxicity have developed.

Table 1 Comparison of Different Acute Liver Failure Etiology Groups

\begin{tabular}{|c|c|c|c|c|c|}
\hline & $\begin{array}{l}\text { APAP } \\
n=787\end{array}$ & $\begin{array}{l}\text { Drug } \\
n=202\end{array}$ & $\begin{array}{l}\text { Indeterminate } \\
n=219\end{array}$ & $\begin{array}{l}\text { НерА/НерВ } \\
n=37 / 123\end{array}$ & $\begin{array}{l}\text { All Others } \\
N=328\end{array}$ \\
\hline Age (median) & 37 & 47 & 38 & $48 / 43$ & 45 \\
\hline Sex (\%F) & 76 & 66 & 60 & $46 / 45$ & 73 \\
\hline Jaundice (days) (median) & 0 & 8 & 8 & $3 / 5$ & 4 \\
\hline Coma $\geq 3(\%)$ & 53 & 37 & 50 & $51 / 55$ & 43 \\
\hline ALT (median) & 3846 & 685 & 849 & $2124 / 1702$ & 677 \\
\hline Bilirubin (median) & 4.4 & 19.8 & 22.0 & $12.5 / 19.1$ & 14.6 \\
\hline $\operatorname{Tx}(\%)$ & 9 & 40 & 45 & $32 / 41$ & 30 \\
\hline Spontaneous survival (\%) & 67 & 31 & 27 & $54 / 24$ & 38 \\
\hline Overall survival (\%) & 75 & 68 & 69 & $84 / 61$ & 65 \\
\hline
\end{tabular}

$N=1696$.

Clinical and demographic features of acute liver failure according to etiologic groups.

Acetaminophen-related acute liver failure (APAP) is considered hyperacute and demonstrates the very high aminotransferase and low bilirubin levels, compared with drug-induced liver injury, which is more indolent. ALT, alanine aminotransferase; TX, transplantation. 


\section{Unintentional Acetaminophen-Related Acute Liver Failure}

The US ALFSG has provided a more detailed snapshot of all acetaminophen-related ALF cases, ${ }^{14}$ with particular attention to the unintentional group. Thirty-eight percent of these patients were simultaneously taking more than one acetaminophen-containing preparation, and 62\% were taking an opioid combination such as hydrocodone and acetaminophen. In many instances, individuals are using alcohol, hypnotics, or illicit drugs in combination, undoubtedly clouding judgment and often delaying hospitalization. In a small number of cases, habituation to the opioid compounds (up to 40 or more tablets per day) may develop over days or weeks prior to onset of liver injury, suggesting addiction to the narcotic component with development of tolerance to the narcotic and to the acetaminophen. Those entering with severe hepatotoxicity have apparently exhausted their compensatory mechanisms. Tolerance has been observed in animals fed increasing acetaminophen doses. ${ }^{15}$ The events prior to presentation are often unclear, but the biochemical picture (AST [aspartate aminotransferase]/ALT elevations/bilirubin levels) in these "chronic" patients is just as acute, suggesting that patients may tolerate both drugs, then experience "breakthrough" for unclear reasons. It is possible that fasting due to an intercurrent illness or simply increasing the dose once too often brings this about. Because patients with unintentional toxicity frequently exhibit signs of polysubstance abuse they may present to the hospital later and are less likely to receive a liver transplant.

As might be expected, suicidal ingestions that eventuate in ALF are associated with late presentation, alcohol or other concomitant drugs that may cloud the sensorium and thus delay presentation, and larger total doses, indicating more serious intent rather than a gesture. Even with late presentation, NAC orally (and now available as an intravenous preparation, Acetadote, Cumberland Pharmaceuticals, Inc., Nashville, TN) is used and provides reliable protection against fatal injury, preventing a large number of deaths when given within 12 hours of ingestion. ${ }^{16}$ Use after 24 hours is recommended, but efficacy cannot be proven for these late ingestions because injury is developed by 36 hours, peaking by 72 hours.

Traditionally, acetaminophen poisoning carries a very good prognosis, even if hepatic failure has developed. However, one third of those reaching the threshold of encephalopathy still die, and only 7 to $9 \%$ undergo transplantation. ${ }^{4}$ The spontaneous survival of the acetaminophen patients who develop encephalopathy (64\%) exceeds that for most other forms of ALF, such as idiosyncratic drug toxicity, where survival without transplantation is only $\sim 20 \%{ }^{14}$ Nevertheless, because of the sheer number of cases, deaths due to acetaminophen toxicity constitute the most frequent cause of death in our study. Suicidal predilection or the history of previous suicide attempts, like substance abuse, will often preclude transplant consideration. Once ALF develops, the outcome for either type of overdose, suicidal or unintentional, is similar.

\section{Other Issues Associated With Acetaminophen}

Acetaminophen cases are all considered hyperacute, with time from onset of jaundice to encephalopathy of 1 day versus idiosyncratic drugs or viral hepatitis where longer intervals are seen. - Table 1 compares presenting and biochemical features of acetaminophen versus idiosyncratic drug reactions and other etiologic categories. As shown, acetaminophen ALF is characterized by very high aminotransferase levels and low bilirubins, whereas much lower aminotransferases and higher bilirubin levels characterize the more slowly evolving pictures.

Recent studies from ALFSG and others highlight more subtle issues surrounding acetaminophen. Using a recently developed assay that reliably detects acetaminophen-containing protein adducts released into the plasma by dying hepatocytes, $20 \%$ of both adult and pediatric ALF patients with indeterminate etiology (no cause discerned after extensive investigation and testing) were found to be due to unrecognized acetaminophen poisoning. ${ }^{17,18}$ Adducts are not found with acetaminophen use unless there is toxicity Elevated adduct levels strongly implicate acetaminophen as the primary cause of the injury (-Fig. 2). The acetaminophen adducts assay provides the smoking gun, evidence of specific hepatocyte damage due to acetaminophen, when historical data are lacking; $\sim 19 \%$ of the indeterminate group have levels of adducts comparable to those observed in suicidal cases, suggesting that a lack of history makes the case indeterminate. ${ }^{19}$ When the biochemical patterns of these indeterminate cases were analyzed, all cases fit the profile of high ALT, low bilirubin observed in the known acetaminophen cases. Insufficient data may result from patient obtundation, or denial, whether purposeful or out of ignorance.

Whether acetaminophen dosing should be limited in the presence of chronic liver disease or cirrhosis remains unclear. A recent paper has questioned the use of $4 \mathrm{~g}$ /day as the standard cutoff for acetaminophen maximum dosing, ${ }^{20}$ and a recent US Food and Drug Administration (FDA) advisory panel recommended lowering the recommended daily dose of $4 \mathrm{~g}$ and "unbundling" the opioid-acetaminophen compounds. To date, the FDA has implemented a change to lower to $325 \mathrm{mg}$ the quantity of acetaminophen allowed in opioid combinations, and the industry is beginning to recommend lower total daily doses. ${ }^{21,22}$

\section{Idiosyncratic Drug Reactions}

The developed world is particularly subject to rare ALF due to idiosyncratic drug-induced liver injury (DILI), because of the large quantity of drugs we ingest. Still, prescription drugs are remarkably safe; the combined number of idiosyncratic drug cases thought to cause ALF in our series is dwarfed by the single agent, acetaminophen. The presentation of DILI is more subacute as shown in $\boldsymbol{-}$ Table $\mathbf{1}$, with lower aminotransferases and higher bilirubin levels. The likelihood of survival in this setting is less than $30 \%$, and they more often undergo liver transplantation. ${ }^{23,24}$ Most frequently implicated drugs include antibiotics (most commonly antituberculous medications, but also sulfa drugs and others). Next most common are nonsteroidal antiinflammatory agents and anticonvulsants.

\section{Viral Hepatitis}

Cases of viral hepatitis that develop hepatic failure represent a small fraction of all cases $(\sim 1 \%)$ and largely consist of 


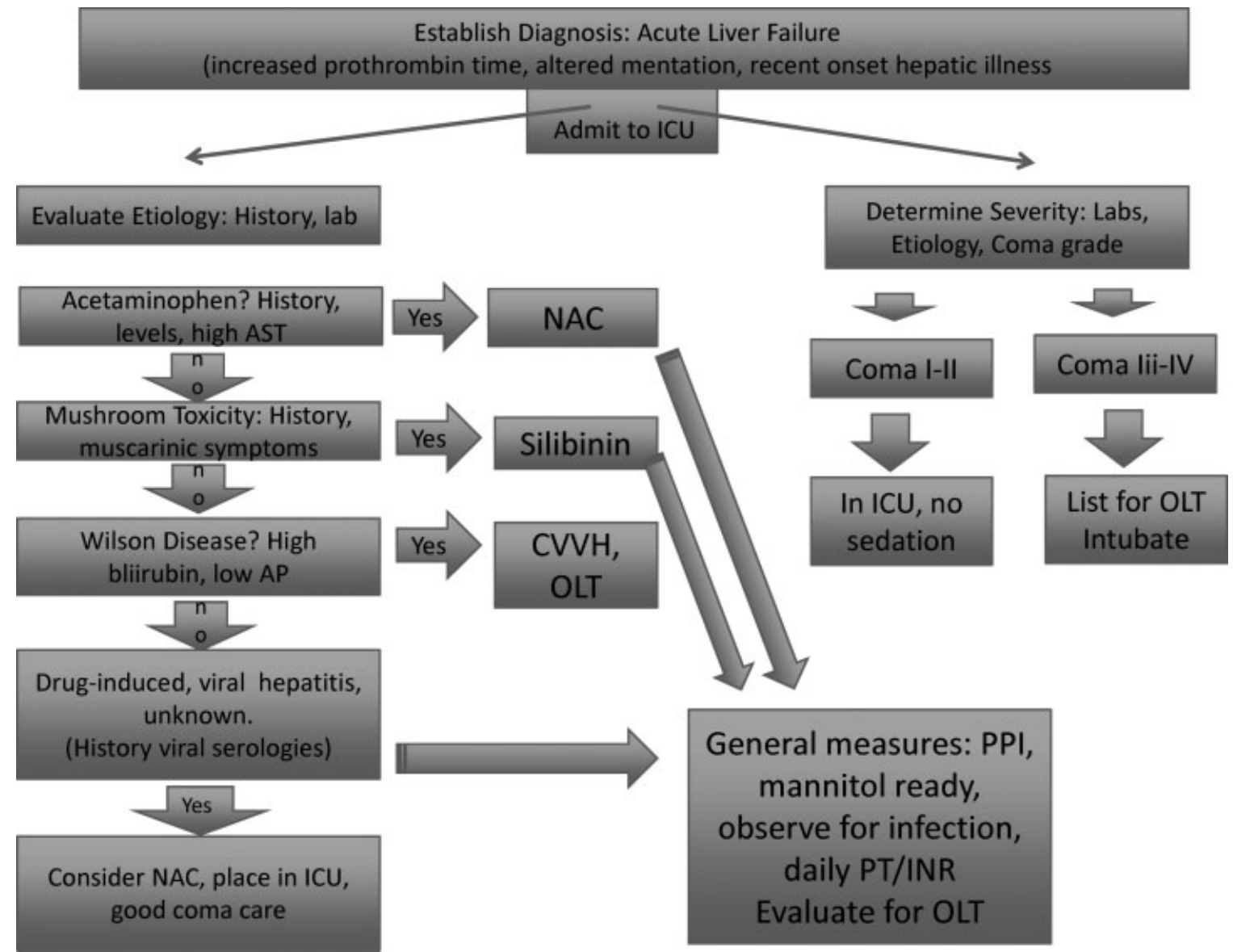

Figure 2 Algorithm for triaging acute liver failure (ALF) patients. The initial step is to establish the diagnosis as ALF, then the etiology, and then the severity. Time is of the essence if transplantation is an option. Patients may rapidly deteriorate, so frequent neurological checks and consideration of intubation are indicated for any significant degree of drowsiness (coma grade 2+ or higher). Sedation is avoided in early coma grades to allow for continued observation of central nervous system function.

hepatitis B, with hepatitis A less frequent and hepatitis E quite prevalent in areas with poor sanitation. Hepatitis B that presents with a fulminant picture may be due to new acute infection or an acute exacerbation of chronic hepatitis B. ${ }^{25}$

\section{Other Causes}

A variety of other etiologies have been implicated from autoimmune hepatitis (which may be difficult to diagnose), ${ }^{26}$ Budd-Chiari syndrome (hepatic vein thrombosis), Wilson disease, ${ }^{27}$ pregnancy-associated liver failure, and the rapid evolution of metastatic or lymphomatous hepatic infiltration. $^{28}$

\section{Indeterminate Cases}

Despite our best efforts, in $15 \%$ of patients the diagnosis eludes us. To date, we have found little evidence for other viruses such as parvovirus B19, hepatitis E, herpesviruses (there are a few cases, but not many), occult hepatitis B, or other previously unrecognized viruses..$^{29,30}$ Unrecognized acetaminophen toxicity is clearly implicated, and there may be unrecognized autoimmune disease as already noted. ${ }^{19,26}$

\section{Clinical Management}

The backbone of management of the ALF patient is good coma care. ${ }^{5,6,31}$ However, some special features deserve mention. Rapid evaluation and initiation of antidotes, where feasible, are needed: NAC for acetaminophen poisoning, penicillin $G$ and silybinin for mushroom poisoning, and delivery of the fetus in the cases of pregnancy-induced ALF are standard of care. Early consideration should be made to use NAC in nonacetaminophen settings. A multicenter, double-blind, placebo-controlled study of its use showed no benefit in overall survival but improved outcomes at 3 weeks and at 1 year for those with early coma grades. ${ }^{32}$ All such trials are compromised by the variety of etiologies and presentations and the rescue provided by hepatic transplantation so that survival of a large number of patients (those receiving liver grafts) is greatly improved, but their true survival without grafting is never known. Only those with ALF are given the most urgent listing by the United Network for Organ Sharing (UNOS). In the NAC study, 50\% of those who were considered to have stage 3 or 4 encephalopathy on admission to study reached an outcome (death or transplant) by study day 4 , 


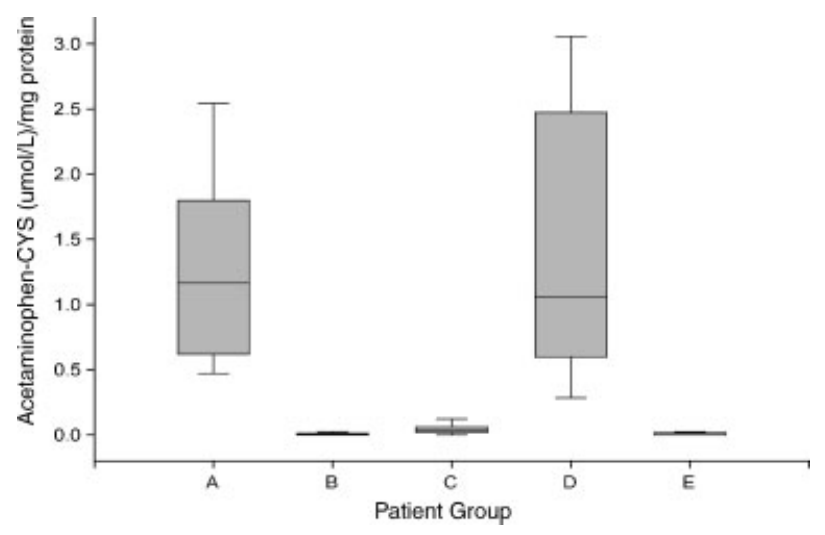

Figure 3 Serum levels of acetaminophen-CYS (cysteine) adducts in patient groups. (A) Patients with acute liver failure (ALF) secondary to known acetaminophen overdose. (B) Patients with ALF owing to nonacetaminophen causes. (C) Patients with acetaminophen overdose but no ALF. (D) Patients with ALF of indeterminate etiology and detectable serum adducts. (E) Patients with ALF of indeterminate etiology and negative adducts. The boxes represent the 25th to 75th IQR (interquartile ranges), and the horizontal line represents the median. The extremes of the population are represented by the end marks. (Reprinted from Davern et al $^{17}$ with kind permission of Elsevier.)

emphasizing the severity of their condition and the availability of transplantation for many patients-40\% ultimately were transplanted. Once the diagnosis of ALF is confirmed (by the finding of coagulopathy and encephalopathy in the setting of acute hepatitis) and a careful search for etiology is undertaken (-Fig. 3), attention should be turned to consideration of the need to list for transplantation. Controversial management issues that are commonly of concern include managing the coagulopathy, whether intracranial pressure (ICP) monitoring is used, and the treatment of cerebral edema. By establishing that the diagnosis is ALF and then determining the etiology and severity-appropriate triage to an intensive care unit or transfer to a transplant center can be rapidly accomplished. Determining eligibility and listing for transplantation must be an early consideration because time is of the essence.

\section{Specific Therapies}

The few therapies that are specific to an etiology emphasize the need for expedient determination of the cause of illness (-Fig. 3). Hepatitis B causing ALF comprises both true acute infection and acute-on-chronic infections. One all too frequent scenario is the reactivation of chronic hepatitis B in the setting of chemotherapy. This is common in the United States but certainly also in Asia. ${ }^{33}$ Nucleoside analogues have been suggested as treatment for ALF due to hepatitis B. In the setting of planned chemotherapy, they are mandated to protect against reactivation. Although very effective as prophylaxis, there is no strong evidence that their use, once ALF develops, improves outcomes. Still, because transplantation is frequently required, nucleoside analogues are routinely used for their posttransplant prophylaxis effect. ${ }^{34}$

\section{Cerebral Edema and the Role of Intracranial Pressure Monitoring}

Cerebral edema is the cause of death in several patients with ALF but the actual percentage is unclear. Development of brain swelling due to osmotic changes within the brain and loss of capillary integrity occur more commonly in hyperacute settings and in young people, where adaptation to rapid changes in brain glutamine levels cannot take place. Thus acetaminophen patients, typically under age 35 , are at highest risk for brain edema. High levels of arterial ammonia have been shown to correlate well with development of cerebral edema, further reinforcing the glutamine hypothesis. ${ }^{35-38} \mathrm{~A}$ retrospective study of the impact of ICP monitoring in $332 \mathrm{ALF}$ patients suggested that use of ICP monitoring was center dependent, reflecting, among other things, the confidence level of the neurosurgeon because a burr hole is needed in the skull and may be hazardous in the presence of severe coagulopathy. Patients in whom a monitor was placed generally received more aggressive mannitol and other treatments when evidence was available clinically of increased ICP levels, and were more likely to be listed for transplantation, but outcomes did not appear to differ in the two groups. ${ }^{39}$

\section{Cardiovascular Tone and Its Management}

Most patients with ALF demonstrate "warm shock"; that is, profound vasodilation and low mean arterial pressures. In addition, due to poor oral intake and encephalopathy prior to admission, most are volume depleted and require initial fluid resuscitation and possibly central venous pressure determinations to aid in this process. Use of pulmonary artery catheters is seldom recommended. The renal hemodynamics resemble those of the patient with hepatorenal syndrome in cirrhosis: low systemic vascular resistance and renal artery vasoconstriction that is potentially reversible with improvement in liver function. Patients with acetaminophen poisoning may demonstrate acute kidney injury due to direct toxic effects of acetaminophen with oliguria requiring continuous venovenous hemodialysis (CVVHD). The initial aim should be volume resuscitation and, if the mean arterial pressure is $<$ $80 \mathrm{~mm} \mathrm{Hg}$, support with pressor agents, the most effective of which appears to be norepinephrine. ${ }^{6}$ In the setting of multiorgan failure, plasma troponin I levels are frequently elevated, but the significance of this finding is unclear. ${ }^{40}$

\section{Role of Coagulopathy in Acute Liver Failure}

Severe alterations in nearly all clotting parameters are seen in ALF in part due to failure of synthesis as well as factor consumption. The classic interpretation has been that severe bleeding is a likely outcome. Overall, however, there are abnormalities in both the coagulation and the fibrinolytic pathways, and recent data suggest that the defects are balanced; that is, there is a relative preservation of hemostasis unless the platelet count is very low. ${ }^{41}$ The reason for low platelet counts has not been elucidated-consumption may play a role, whereas thrombopoietin is also made within the liver. Thrombopoietin levels are not uniformly low and do not appear to correlate with platelet levels. ${ }^{42}$ In general, fresh frozen plasma (FFP), cryoprecipitate, or platelets are reserved 
for active bleeding or if an invasive procedure is planned. There is little support for the use of recombinant factor VIIa in this setting. Withholding FFP allows for the use of the INR as a continuously available and reliable measure of hepatic synthetic function and outcome.

\section{Prognosis}

Determining prognosis in ALF is vital. Unlike cirrhosis, use of a transplant in ALF would be unnecessary if the patient can fully recover without a graft. If transplanted, lifelong immunosuppression is also the dividend. Prognostic scores specific for ALF have been limited in efficacy; the most commonly utilized is the King College Criteria (KCC). ${ }^{43}$ These and other scoring systems to date have been relatively sensitive but not specific, with fairly robust prediction of death but inability to reliably determine who will survive. This may be because more patients could recover if complications such as infection did not occur. Two key factors involved in determining outcome are etiology and coma grade on admission (-Figs. 3 and $\mathbf{4}>$ ). Acetaminophen, hepatitis A, ischemia, and pregnancy are all associated with at least $60 \%$ short-term survival without transplantation, whereas DILI, hepatitis B, autoimmune hepatitis, and indeterminate causes are associated with $\sim 30 \%$ spontaneous survival. Presenting with early coma grade allows for a much more favorable outcome prediction across all etiologies (-Fig. 4).

\section{Role of Transplantation}

There is no fully proven medical therapy for this devastating clinical syndrome. Prior to 1980, ALF series demonstrated survival in less than $10 \%$. Small series of cases subjected to new experimental treatments (e.g., heparin) showed that occasionally patients do survive; however, controlled trials were virtually impossible to perform. In this setting, trans- plantation seemed nothing short of a miracle. In the current era, overall survival is $\sim 67 \%$, a considerable improvement in the last 3 decades. However, transplants are only performed on 25 to $30 \%$ of patients admitted to the ALF study (-Fig. 5 ). The reasons for the relatively infrequent use of liver grafting include the difficulties in obtaining organs in an urgent fashion, as well as the changing mix of etiologies. The change from hepatitis B to a predominance of acetaminophen makes up much of these differences because acetaminophen clearly carries a better prognosis than most other etiologies and is often associated with compromising psychosocial issues and substance abuse.

Even in the transplant era, nearly $30 \%$ of patients die with ALF. Reasons for the failure to transplant more patients have included lack of available organs, late presentation with rapid deterioration, substance abuse issues, repeated suicidal behavior, or other organ system involvement (malignancy, heart failure) that would preclude grafting. Patients undergoing transplantation for ALF are typically young and otherwise healthy and thus should be quite optimal candidates compared with their cirrhosis counterparts. Nevertheless, shortterm and 1-year survival figures are below those for cirrhotic patients, in part because of the extreme emergency conditions often encountered. ${ }^{44}$ There is no option for deferring transplant in these patients. Similarly, living-related donors might solve the organ shortage; however, it is extremely difficult to prepare a donor adequately and perform the surgery in a timely fashion. Few living related donors have been used to date in adults with ALF, although this has gained some traction in pediatric ALF where the graft size needed can be quite small. ${ }^{45}$ Despite full physical recovery, many patients who have experienced cerebral edema have mental impairment that is lifelong. To date, few long-term data on these complications are available.

\section{Transplant-free survival by etiology and coma grade}

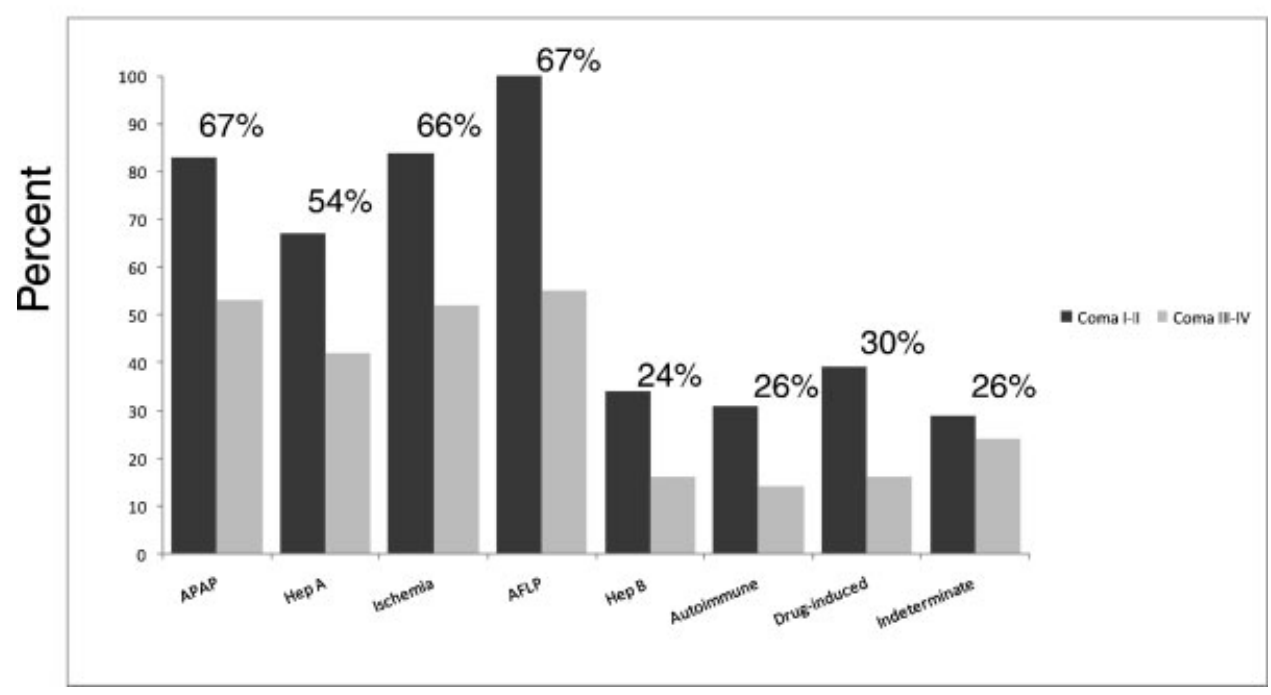

Figure 4 Outcomes according to etiology and coma grade. The bars for each etiology indicate the percent survival without transplantation according to coma grade (I, II vs III, IV). The percent above each pair indicates the overall percentage of spontaneous survival for that etiology. There is a disparity between the etiologies with good survival and those with poor survival, but survival is much poorer in all categories if the patient is admitted with an advanced coma grade. 


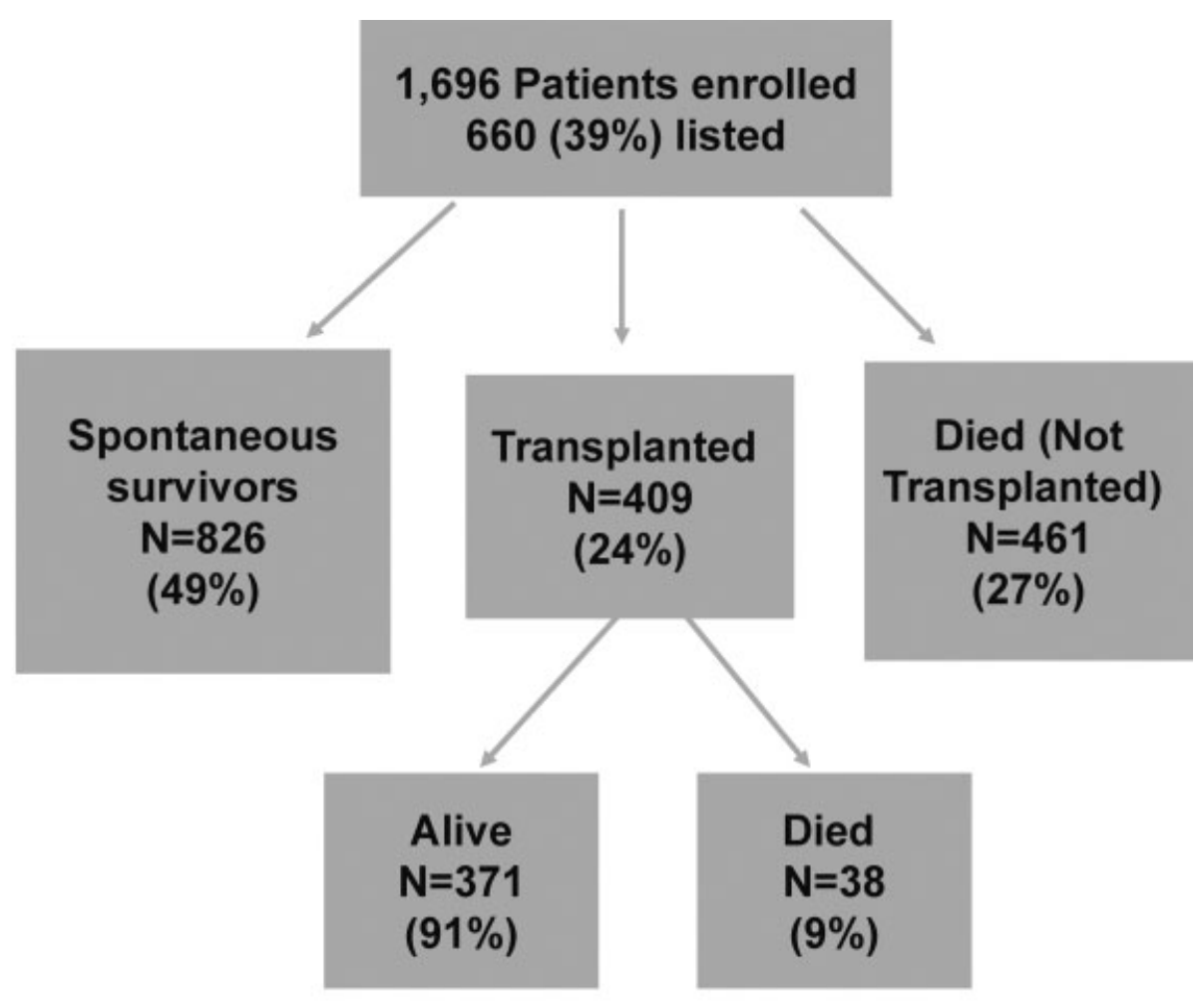

\section{Overall survival: $\mathrm{N}=1,197(71 \%)$}

Figure 5 Outcomes for 1696 patients enrolled in the Acute Liver Failure Study Group study. Only 39\% are listed for transplantation, and 24\% receive a graft.

\section{New Therapies Undergoing Current Trial}

To date, the NAC trial is one of the very few controlled trials in ALF, and its results remain controversial. ${ }^{46}$ More recent efforts have been directed toward reliable means to lower ammonia levels. Neither lactulose nor rifaximin has been shown to favorably affect outcomes or coma grade in ALF, but they are still widely used. Surgeons discourage the use of lactulose because it may lead to gaseous distension of the colon, interfering with access to the surgical field. A blinded, controlled trial performed in India sought to test the value of L-ornithine L-acetate infusions in 203 patients with ALF, a substance that would alter the balance of glutamate toward glutamine in the circulation thus trapping ammonia, based on animal studies. ${ }^{47}$ There appeared to be no benefit in patients either in lowering ammonia or in improving outcomes, perhaps because ammonia is regenerated by gut glutaminases. ${ }^{48} \mathrm{~A}$ new compound, ornithine phenyl acetate, is currently under consideration after several trials in experimental animals. ${ }^{49,50}$

Hypothermia to protect brain function has also been demonstrated in animals and humans to reliably lower ICP. It is not routinely used at present, in part due to uncertainties regarding its safety. The challenging logistics of performing clinical trials in patients with ALF to evaluate hypothermia has thus far defeated efforts to demonstrate its safety or efficacy.

\section{Bio-Artificial Liver Assist Devices}

An artificial liver device that would replace the diverse functions of the liver is an unmet need that seems far in the future. Several groups have designed membrane-containing cartridges filled with hepatocytes from human cell lines or animal donors, allowing plasma to flow by the cells. Some improvement in encephalopathy has been observed, but no real improvement could be demonstrated in overall survival in a controlled trial. ${ }^{51,52} \mathrm{~A}$ recent meta-analysis further demonstrated the scarcity of data and the lack of convincing efficacy. ${ }^{53}$ Several centers have experimented with extracorporeal transgenic pig livers or cadaver livers deemed unfit for transplantation, but these techniques have not gained general acceptance. ${ }^{54,55}$ Finally, early experimentation is under way using infusions of hepatic stem cells that are said to be nonimmunogenic ${ }^{56}$; this remains highly experimental.

\section{Summary}

ALF challenges our best clinical and surgical skills because of its rarity, rapid progression, and frequently poor outcomes. The small numbers of patients do not readily lend themselves to controlled trials and are studied only with great difficulty. Nevertheless, descriptive information aids our understanding of what to expect and where small gains might be made in 
this condition. Patients are particularly vulnerable to infection, bleeding, and cerebral edema and seek medical care typically with very advanced hepatic injury. It is unlikely that there is one overall therapy that will improve hepatic function and restore hepatocyte mass in this condition. Rescue therapies that provide temporary liver support, or other treatments short of transplantation that don't enhance hepatic regeneration, are likely to fail unless there is reconstitution of functional hepatic stem cells. Most of our efforts in treating ALF in 2011 should be directed toward ameliorating the damage in various etiologic categories. Possible targets might include, for acetaminophen, further education regarding the risk of acetaminophen causing liver injury, and limitation of exposure for over-the-counter use.

\section{References}

1 Trey C, Davidson CS. The management of fulminant hepatic failure. In: Popper H, Schaffner F. eds. Progress in Liver Diseases. New York: Grune \& Stratton; 1970:282-298

2 Lee WM, Squires RH Jr, Nyberg SL, Doo E, Hoofnagle JH. Acute liver failure: summary of a workshop. Hepatology 2008;47(4):14011415

3 Bernal W, Auzinger G, Dhawan A, Wendon J. Acute liver failure. Lancet 2010;376(9736):190-201

4 Ostapowicz GA, Fontana RJ, Schiødt FV, et al; U.S. Acute Liver Failure Study Group. Results of a prospective study of acute liver failure at 17 tertiary care centers in the United States. Ann Intern Med 2002;137(12):947-954

5 Stravitz RT, Kramer AH, Davern T, et al; Acute Liver Failure Study Group. Intensive care of patients with acute liver failure: recommendations of the U.S. Acute Liver Failure Study Group. Crit Care Med 2007;35(11):2498-2508

6 Stravitz RT, Kramer DJ. Management of acute liver failure. Nat Rev Gastroenterol Hepatol 2009;6(9):542-553

7 Lee WM, Schiødt FV. Fulminant hepatic failure. In: Schiff ER, Sorrell MF, Maddrey WCeds. Schiff's Diseases of the Liver. 8th ed. Philadelphia: Lippincott-Raven; 1999:879-895

8 Schiødt FV, Atillasoy E, Shakil AO, et al. Etiology and outcome for 295 patients with acute liver failure in the United States. Liver Transpl Surg 1999;5(1):29-34

9 Lee WM, Seremba E. Etiologies of acute liver failure. Curr Opin Crit Care 2008;14(2):198-201

10 Acharya SK, Dasarathy S, Kumer TL, et al. Fulminant hepatitis in a tropical population: clinical course, cause, and early predictors of outcome. Hepatology 1996;23(6):1448-1455

11 Zimmerman HJ, Maddrey WC. Acetaminophen (paracetamol) hepatotoxicity with regular intake of alcohol: analysis of instances of therapeutic misadventure. Hepatology 1995;22(3):767773

12 Shakil AO, Kramer D, Mazariegos GV, Fung JJ, Rakela J. Acute liver failure: clinical features, outcome analysis, and applicability of prognostic criteria. Liver Transpl 2000;6(2):163-169

13 Schiødt FV, Rochling FA, Casey DL, Lee WM. Acetaminophen toxicity in an urban county hospital. N Engl J Med 1997;337 (16):1112-1117

14 Larson AM, Polson J, Fontana RJ, et al; Acute Liver Failure Study Group. Acetaminophen-induced acute liver failure: results of a United States multicenter, prospective study. Hepatology 2005;42;1367-1372

15 Shayiq RM, Roberts DW, Rothstein K, et al. Repeat exposure to incremental doses of acetaminophen provides protection against acetaminophen-induced lethality in mice: an explanation for high acetaminophen dosage in humans without hepatic injury. Hepatology 1999;29(2):451-463
16 Larson AM. Acetaminophen hepatotoxicity. Clin Liver Dis 2007;11 (3):525-548, vi

17 Davern TJ II, James LP, Hinson JA, et al; Acute Liver Failure Study Group. Measurement of serum acetaminophen-protein adducts in patients with acute liver failure. Gastroenterology 2006;130 (3):687-694

18 James LP, Alonso EM, Hynan LS, et al; Pediatric Acute Liver Failure Study Group. Detection of acetaminophen protein adducts in children with acute liver failure of indeterminate cause. Pediatrics 2006;118(3):e676-e681

19 Khandelwal N, James LP, Sanders C, Larson AM, Lee WM; and the Acute Liver Failure Study Group. Unrecognized acetaminophen toxicity as a cause of indeterminate acute liver failure. Hepatology 2011;53(2):567-576

20 Watkins PB, Kaplowitz N, Slattery JT, et al. Aminotransferase elevations in healthy adults receiving 4 grams of acetaminophen daily: a randomized controlled trial. JAMA 2006;296(1):87-93

21 U.S. Food and Drug Administration. FDA drug safety communication: prescription acetaminophen products to be limited to $325 \mathrm{mg}$ per dosage unit: boxed warning will highlight potential for severe liver failure. http://www.fda.gov/Drugs/DrugSafety/ ucm239821.htm. Accessed August 21, 2011

22 Harris G. F.D.A. plans new limits on painkillers. New York Times. January 13, 2011. http://www.nytimes.com/2011/01/14/health/ policy $/ 14 \mathrm{fda} . \mathrm{html}$ ?scp=1\&sq=tylenol\%20package\&st=cse. Accessed August 21, 2011

23 Chalasani N, Fontana RJ, Bonkovsky HL, et al; Drug Induced Liver Injury Network (DILIN). Causes, clinical features, and outcomes from a prospective study of drug-induced liver injury in the United States. Gastroenterology 2008;135(6):1924-1934, e1-e4

24 Reuben A, Koch DG, Lee WM; Acute Liver Failure Study Group. Drug-induced acute liver failure: results of a U.S. multicenter, prospective study. Hepatology 2010;52(6):2065-2078

25 Dao DY, Hynan LS, Yuan HJ, et al; The Acute Liver Failure Study Group. Two distinct subtypes of hepatitis B virus-related acute liver failure are separable by quantitative serum IgM anti-HBc and HBV DNA levels. Hepatology 2011 Oct 10. doi:10.1002/hep24732. [Epub ahead of print]

26 Stravitz RT, Lefkowitch JH, Fontana RJ, et al; Acute Liver Failure Study Group. Autoimmune acute liver failure: proposed clinical and histological criteria. Hepatology 2011;53(2):517-526

27 Korman JD, Volenberg I, Balko J, et al; Pediatric and Adult Acute Liver Failure Study Groups. Screening for Wilson disease in acute liver failure: a comparison of currently available diagnostic tests. Hepatology 2008;48(4):1167-1174

28 Rowbotham D, Wendon J, Williams R. Acute liver failure secondary to hepatic infiltration: a single centre experience of 18 cases. Gut 1998;42(4):576-580

29 Lee WM, Brown KE, Young NS, et al; Acute Liver Failure Study Group. Brief report: no evidence for parvovirus B19 or hepatitis E virus as a cause of acute liver failure. Dig Dis Sci 2006;51 (10):1712-1715

30 Levitsky J, Duddempudi AT, Lakeman FD, et al; US Acute Liver Failure Study Group. Detection and diagnosis of herpes simplex virus infection in adults with acute liver failure. Liver Transpl 2008;14(10):1498-1504

31 Polson J, Lee WM; American Association for the Study of Liver Disease. AASLD position paper: the management of acute liver failure. Hepatology 2005;41(5):1179-1197

32 Lee WM, Hynan LS, Rossaro L, et al; Acute Liver Failure Study Group. Intravenous N-acetylcysteine improves transplant-free survival in early stage non-acetaminophen acute liver failure. Gastroenterology 2009;137(3):856-864, e1

33 Oketani M, Ido A, Tsubouchi H. Changing etiologies and outcomes of acute liver failure: a perspective from Japan. J Gastroenterol Hepatol 2011;26(Suppl 01):65-71 
34 Sorrell MF, Belongia EA, Costa J, et al. National Institutes of Health consensus development conference statement: management of hepatitis B. Ann Intern Med 2009;150(2):104-110

35 Blei AT. Pathogenesis of brain edema in fulminant hepatic failure. Prog Liver Dis 1995;13:311-330

36 Jalan R. Pathophysiological basis of therapy of raised intracranial pressure in acute liver failure. Neurochem Int 2005;47(1-2):78-83

37 Larsen FS, Wendon J. Prevention and management of brain edema in patients with acute liver failure. Liver Transpl 2008;14(Suppl 02):S90-S96

38 Clemmesen JO, Larsen FS, Kondrup J, Hansen BA, Ott P. Cerebral herniation in patients with acute liver failure is correlated with arterial ammonia concentration. Hepatology 1999;29(3): 648-653

39 Vaquero J, Fontana RJ, Larson AM, et al. Complications and use of intracranial pressure monitoring in patients with acute liver failure and severe encephalopathy. Liver Transpl 2005;11 (12):1581-1589

40 Parekh NK, Hynan LS, De Lemos J, Lee WM; Acute Liver Failure Study Group. Elevated troponin I levels in acute liver failure: is myocardial injury an integral part of acute liver failure? Hepatology 2007;45(6):1489-1495

41 Stravitz RT, Lisman T, Luketic VA, et al. Minimal effects of acute liver injury/acute liver failure on hemostasis as assessed by thromboelastography. J Hepatol 2012;56(1):129-136

42 Schiødt FV, Balko J, Schilsky MC, Harrison ME, Thornton A, Lee WM; Acute Liver Failure Study Group. Thrombopoietin in acute liver failure. Hepatology 2003;37(3):558-561

43 O'Grady JG, Alexander GJ, Hayllar KM, Williams R. Early indicators of prognosis in fulminant hepatic failure. Gastroenterology 1989;97(2):439-445

44 Lee WM, Squires RH Jr, Nyberg SL, Doo E, Hoofnagle JH. Acute liver failure: summary of a workshop. Hepatology 2008;47(4):14011415

45 Shouval DS, Mor E, Avitzur Y, et al. Living-related donor liver transplantation for children with fulminant hepatic failure in Israel. J Pediatr Gastroenterol Nutr 2009;48(4):451-455
46 Hanje AJ, Michaels A, Lee WM. Is $N$-acetylcysteine effective in all non-acetaminophen acute liver failure? In: Jensen DM, ed. Controversies in Hepatology. Philadelphia, PA: Slack; 2011

47 Acharya SK, Bhatia V, Sreenivas V, Khanal S, Panda SK. Efficacy of Lornithine L-aspartate in acute liver failure: a double-blind, randomized, placebo-controlled study. Gastroenterology 2009;136 (7):2159-2168

48 Jalan R, Lee WM. Treatment of hyperammonemia in liver failure: a tale of two enzymes. Gastroenterology 2009;136(7):2048-2051

49 Davies NA, Wright G, Ytrebø LM, et al. L-ornithine and phenylacetate synergistically produce sustained reduction in ammonia and brain water in cirrhotic rats. Hepatology 2009;50(1):155-164

50 Ytrebø LM, Kristiansen RG, Maehre H, et al. L-ornithine phenylacetate attenuates increased arterial and extracellular brain ammonia and prevents intracranial hypertension in pigs with acute liver failure. Hepatology 2009;50(1):165-174

51 Demetriou AA, Brown RS Jr, Busuttil RW, et al. Prospective, randomized, multicenter, controlled trial of a bioartificial liver in treating acute liver failure. Ann Surg 2004;239(5):660-667, discussion 667-670

52 Jalan R, Sen S, Williams R. Prospects for extracorporeal liver support. Gut 2004;53(6):890-898

53 Liu J, Kjaergard LL, Als-Nielsen B, Gluud C. Artificial and bioartificial support systems for liver failure: a Cochrane Hepato-Biliary Group Protocol. Liver 2002;22(5):433-438

54 Chari RS, Collins BH, Magee JC, et al. Brief report: treatment of hepatic failure with ex vivo pig-liver perfusion followed by liver transplantation. N Engl J Med 1994;331(4):234-237

55 Horslen SP, Hammel JM, Fristoe LW, et al. Extracorporeal liver perfusion using human and pig livers for acute liver failure. Transplantation 2000;70(10):1472-1478

56 Fukumitsu K, Yagi H, Soto-Gutierrez A. Bioengineering in organ transplantation: targeting the liver. Transplant Proc 2011;43 (6):2137-2138 